\title{
PEMBUATAN LAPISAN TIPIS DAN SERBUK TIMBAL TITANAT DENGAN METODA SOL GEL
}

\author{
Rahmayeni, Emriadi, Eli Susanti, dan Delfi Silvia \\ Jurusan Kimia, FMIPA, Universitas Andalas, Padang
}

\begin{abstract}
Thin layer and powder of lead titanate had been prepared using lead acetate and tetraethylorthotitanate as precursor by sol gel process. The thin layer of composite was obtain through layering the composite on glass substrate. Both thin layer and powder were calcined between $100-700^{\circ} \mathrm{C}$ and were characterized by Scanning Electron Microscopy (SEM), X-ray diffraction (XRD), UV and Fourier Transform Infra Red (FTIR). The diffraction pattern show that the structure of composite on glass substrate is amorf at $500^{\circ} \mathrm{C}$ and could not identified at $700^{\circ} \mathrm{C}$ while the powder is crystalline at 500 and $700^{\circ} \mathrm{C}$. FTIR spectra shown the Ti-O peak at $1407 \mathrm{~cm}^{-1}$, the $\mathrm{Pb}-\mathrm{O}$ and $\mathrm{Pb}-\mathrm{O}-\mathrm{Ti}$ peaks at 718 and $600 \mathrm{~cm}^{-1}$, respectively.
\end{abstract}

\section{DAFTAR PUSTAKA}

1. Camargo, E. R., Kakihana, M., 2001, Peroxide - Based Route Free from Halides for the Synthesis of Lead Titanat Powder, J.Chem. Mater., 13: 1181-1184.

2. Li, Z., Wu, A., and Vilarinho, P.M., 2004, Perovskite Phase Stabilization Of $\mathrm{Pb}\left(\mathrm{Zn}_{1 / 3} \mathrm{Ta}_{2 / 3}\right) \mathrm{O}_{3}$ Ceramics Induced by $\mathrm{PbTiO}_{3}$ Seed, J. Chem. Mater., 16: 718719.

3. Bosak, A.A., Samoilenkov, S.V., Gorbenko, O.Y., Graboy, I.E., Botev, A.N., Kaul, A.R., 2001, Self-tuning MOCVD Approach to the Growth of Very Smooth $\mathrm{La}_{1-\mathrm{x}} \mathrm{Pb}_{\mathrm{x}} \mathrm{MnO}_{3}$ and $\mathrm{PbTiO}_{3}$ Epitaxial Thin Films, J. Chem. Mater., 13: 981-986.

4. Tang, X.G., Ding, A.L., Ye, Y., and Chan, H.L.W., 2002, Preparation and Characterization of Lead Zirconate Thin Films by Chemical Solution Deposition, $J$. Chem. Mater., 14: 2129.

5. Briggle, J., Petuskey, W.T., Shemkunas, M., 2000, Perovskite Electroceramics. Departement of Chemistry, Arizona State University, Materials Research Science and Engineering Centre (MRSEC). Winter Workshop, January.
6. Schwartz, R.W., 1997, Chemical Solution Deposition of Perovskite Thin Films, J. Chem.Mater., 9: 2325-2340.

7. Brinker, C. J., Hund, A.J., 1990, Fundamental of Sol-Gel Dip-Coating. SolGel Science and Technology, 158-159.

8. Seveno, R., Limousine, P., Averty, D., Chartier, J-L., Le Bihan, R., Gundel, H.W., 2000, Preparation of Multi-Coating PZT Thick Films by Sol-Gel Method onto Stainless Steel Substrates, J. European Ceramic Society., 20: 2025-2028.

9. Jamarun, N., 2000, Proses Sol-Gel. Jurusan Kimia Fakultas Matematika dan Ilmu Pengetahuan Alam Universitas Andalas Padang, hal. 1-23.

10. Fang, J., Wang, J., Ming, L., Ng, S.C., 2002, Comparative Study on Phase Development of Lead Titanate Powders, $J$. Mat. Letters., 52: 304-312.

11. Fang, J., Wang, J., Ming, L., Ng, S.C., 2002, J. Mat. Letters, 52, 304-312.

12. Smith, A.L., 1979, Applied Infrared Spectroscopy, A.Wiley-Interscience Publication, Jhon Wiley \& Sons, Vol. 54, 184-185.

13. Sibilia, J.P., 1996, A Guide to Materials Characterization and Chemical AnalysisI. Wiley-VCH USA, Inc. $2^{\text {nd }}$ Edition, 17-21, 143-147, 167-171. 
